\title{
Cola millenii K. Schum : Etat des connaissances et perspectives de recherche
}

\author{
Iboukoun Fidèle LAWIN ${ }^{1,2 *}$, Adandé Belarmain FANDOHAN ${ }^{2,3,4}$, Kisito GANDJI ${ }^{4}$, \\ Achille Ephrem ASSOGBADJO ${ }^{2,4}$ et Christine Adjokè Ifètayo Nougbodé OUINSAVI ${ }^{1}$ \\ ${ }^{I}$ Laboratoire d'Etudes et de Recherches Forestières (LERF), Faculté d'Agronomie, Université de Parakou, BP \\ 123 Parakou, Bénin. \\ ${ }^{2}$ Laboratoire d'Ecologie Appliquée (LEA), Faculté des Sciences Agronomiques, Université d'Abomey-Calavi, \\ 01 BP 526 Cotonou, Bénin. \\ ${ }^{3}$ Unité de Recherche en Foresterie, Agroforesterie et Biogéographie, Ecole de Foresterie et Ingénierie du Bois, \\ Université Nationale d'Agriculture, BP 43, Kétou, Bénin. \\ ${ }^{4}$ Laboratoire de Biomathématiques et d'Estimations Forestières (LABEF), Faculté des Sciences Agronomiques, \\ Université d'Abomey-Calavi, 04 BP 1525, Cotonou, Bénin. \\ *Auteur correspondant ; E-mail : ifilawin@yahoo.fr; Tél. : (+229) 95114093
}

\section{RESUME}

Cola millenii est un fruitier sauvage comestible peu connu, négligé et sous utilisé en Afrique de l'Ouest. La présente synthèse littéraire a pour objectif de faire le point des connaissances sur l'espèce et de relever les axes de recherche-développement. Les moteurs de recherche Google Scholar et Science Direct ont été utilisés pour la recherche de littérature sur l'espèce avec des mots-clés pertinents. Des publications évaluées par les pairs, livres, thèses et mémoires obtenus dans diverses bibliothèques ont été également consultés. Il ressort de l'analyse de la littérature que $C$. millenii est une plante à usages multiples qui est retrouvée dans plusieurs pays. Elle renferme beaucoup d'éléments minéraux et de la vitamine $C$ nécessaires aux besoins nutritionnels de la population ainsi que des composés chimiques tels qu'alcaloïdes, tannins, saponines, glycosides cardiaques, glucides, stérols, résine et terpènes responsables de son action pharmacologique. En vue de sa conservation et domestication, des travaux complémentaires méritent d'être entrepris sur les variations des usages, l'écologie, la morphologie, la caractérisation biochimique et la phénologie de l'espèce.

(c) 2018 International Formulae Group. All rights reserved.

Mots clés : Cola millenii, fruitiers sauvages comestibles, produits forestiers non ligneux, plante médicinale.

\section{Cola millenii K. Schum: State of knowledge and research prospects}

\begin{abstract}
Cola millenii is a little known, neglected and underutilized wild edible fruit tree in West Africa. The aim of this bibliographic synthesis is to take stock of the knowledge of the species and to highlight the research and development areas. Search engines Google Scholar and Science Direct were used for literature review on this species with relevant keywords. Peer reviewed publications, books, theses and dissertations obtained from various libraries were also consulted. The analysis of the literature shows that $C$. millenii is a multipurpose plant found in several countries. It contains many mineral elements and vitamin $\mathrm{C}$ necessary for nutritional needs of the population as well as chemical compounds such as alkaloids, tannins, saponins, cardiac
\end{abstract}


glycosides, carbohydrates, sterols, resin and terpenes responsible for its pharmacological action. In view of its conservation and domestication, further research endeavors could tackle patterns of uses, ecology, morphology, biochemical characterization and phenology of the species.

(C) 2018 International Formulae Group. All rights reserved.

Keywords: Cola millenii, wild edible fruit trees, non-timber forest products, medicinal plant.

\section{INTRODUCTION}

En Amérique latine, en Asie et en Afrique, plusieurs travaux se sont intéressés aux ressources forestières ainsi qu'à la dépendance des populations vis-à-vis de celles-ci (Codjia et al., 2003). Ces travaux ont montré avec éloquence que partout dans le monde, et notamment chez les communautés rurales, les plantes sont quotidiennement prélevées par les populations pour des usages domestiques ou à des fins commerciales (Kokou et al., 2013). Une gamme importante d'espèces ligneuses pérennes réparties dans les régions tropicales humides permet aux populations indigènes de satisfaire leurs besoins (FAO, 2004). Ces ressources végétales forestières alimentaires qui donnent des fruits, des graines, des tubercules, des fleurs, des sèves et autres produits comestibles contribuent directement à l'alimentation et à l'économie des ménages ruraux (AvocèvouAyisso et al., 2009; Gouwakinnou et al., 2011; Dan Guimbo et al., 2012 ; Houéssou et al., 2012 ; Gbesso 2014 ; Houètchégnon et al., 2015; Makalao et al., 2015 ; Sourou et al., 2016). Elles peuvent donc jouer un rôle important dans les programmes de diversification agricole et de développement de l'agroforesterie en Afrique (Assogbadjo et al., 2012). Cola millenii K. Schum est l'une des ressources phytogénétiques de grandes importances pour les communautés rurales en Afrique de l'Ouest. C'est une espèce forestière à fruits comestibles mais peu connu, négligé et sous utilisé. Au Bénin, ses peuplements naturels sont soumis à des pressions dues à une agriculture itinérante sur brûlis, l'exploitation forestière illicite et la dégradation des berges fluviales entre autres (Dansi et al., 2009). En vue de faciliter son intégration dans les politiques formelles de restauration de ses habitats naturels, de domestication et de sylviculture, il urge de faire le point sur l'état des connaissances de l'espèce et la lumière sur les aspects encore peu élucidés.

La présente étude avait donc pour objectif de faire une synthèse bibliographique sur les informations existantes (taxonomie et systématique; description botanique; origine, écologie et distribution géographique; appellations locales, utilisations et importance économique; propriétés chimiques; propagation et génétique; insectes nuisibles, menaces anthropiques et conservation) sur l'espèce et d'identifier les axes éventuels de recherche et développement.

\section{PROTOCOLE DE COLLECTE ET D'ANALYSE DES DONNEES}

En un premier temps, les deux premiers auteurs du présent travail ont effectué séparément des recherches documentaires à travers l'introduction des mots clés dans les moteurs de recherche Google Scholar et Science Direct. Les principaux mots clés utilisés sont: Cola millenii; écologie + Cola millenii; nutriment + Cola millenii; anti-nutriment + Cola millenii; activité antioxydante + Cola millenii; activité antimicrobienne + Cola millenii; composition proximale + Cola millenii; vitamine + Cola millenii. Cette première phase a abouti à l'identification de plus de 8000 documents. Une première analyse sommaire a été effectuée et il a été convenu de ne prendre en compte que les publications contenant le binôme latin "Cola millenii" et/ou "Cola togoensis" (synonyme). Ce qui a réduit le nombre de documents à analyser en profondeur à 286. Parmi ces documents, tous ceux libres d'accès ont été téléchargés et pris en compte dans l'analyse si les informations véhiculées sont jugées pertinentes pour le 
manuscrit par l'ensemble des auteurs. Pour les publications non libre d'accès, la base AGORA a été consultée en utilisant le code d'accès institutionnel du laboratoire d'appartenance du deuxième auteur. Toutes les publications contenant "Cola millenii" dans la base AGORA ont été téléchargées et scrutée pour prise en compte dans le manuscrit. Des livres, thèses et mémoires obtenus dans diverses bibliothèques ont été également consultés. Au total, 92 documents divers ont été pris en compte dans le cadre de cette analyse bibliographique. Les informations recueillies ont été analysées et présentées sous forme de tableaux et figures.

\section{TAXONOMIE}

Cola est un genre d'environ 125 espèces d'arbres indigènes de l'Afrique tropicale (Ratsch, 2005). Il est représenté par sept (7) taxons au Bénin (Akoègninou et al., 2006). Il s'agit de C. acuminata (P.Beauv.) Schott \& EndI., C. gigantea A. Chev. var. gigantea, $C$. gigantea A. Chev. var. glabrescens Brenan \& Keay, C. lateritia K. Schum, C. laurifolia Mast., C. millenii K. Schum et $C$. nitida (Vent.) Sebott \& EndI. La position systématique de $C$. millenii se présente comme suit :

Règne : Végétal

Embranchement : Spermaphyte

Sous-embranchement : Angiospermes

Classe : Dicotylédones

Ordre : Malvales

Famille : Malvaceae

Sous-famille : Sterculioideae

Genre : Cola

Espèce : Cola millenii

\section{DESCRIPTION BOTANIQUE}

C. millenii est un arbre pouvant atteindre $18 \mathrm{~m}$ de hauteur (Hutchinson et Dalziel, 1958 ; Akoègninou et al., 2006), voire $20 \mathrm{~m}$, mais rarement (Adegoke et al., 1968 ; Nkongmeneck, 1982 ; Burkill, 2004) avec un fût atteignant $50 \mathrm{~cm}$ de diamètre (Burkill, 2004) (Figure1). Les feuilles sont minces et lobées (Figure 2a) (Akoègninou et al., 2006). Les lobes sont faiblement acuminés. Les inflorescences en cymes sont très courtes et axillaires. Les fleurs sont courtement pédicellées avec des sépales verdâtres à l'extérieur, rouge brun ou pourpres à l'intérieur (Figure 2b) (Akoègninou et al., 2006). Les fruits sont constitués de follicules rouges (Figure 2c) (Adjanohoun et al., 1989), roseriche ou rouge-orange (Adebola et Morakinyo, 2006), disposés en étoiles à rayons courts (Figure 2c) plus ou moins nombreux au sommet d'un pédoncule commun et contenant plusieurs graines (Adjanohoun et al., 1989). Les graines (Figure 2d) sont enfouies dans une pulpe blanchâtre (Arbonnier, 2004) (Figure 2e) acidulée (Houenon, 1980) et sucrée (Arbonnier, 2004). L'écorce est fibreuse (Figure 2f) et le bois est blanc et tendre (Hutchinson et Dalziel, 1958).

\section{ORIGINE, ÉCOLOGIE ET DISTRIBUTION GÉOGRAPHIQUE}

Originaire de l'Afrique de l'Ouest (Russell, 1955), C. millenii est une espèce de forêt dense humide semi-décidue (Akoègninou et al., 2006 ; Aoudji et Ganglo, 2006 ; Adjakpa et Akpo, 2008; Adjakpa et al., 2011; Alohou et al., 2017) et forêt marécageuse (Akoègninou et al., 2006 ; Dan, 2009 ; Adjakpa et al., 2011) rencontrée de la Côte d'Ivoire au Nigéria (Akoègninou et al., 2006; Adekunle et Olagoke, 2008). Elle se trouve aussi en forêt galerie (Houenon, 1980 ; Natta, 2003 ; Ganglo et de Foucault, 2006 ; Ceperley et al., 2010; Adjakpa et al., 2013; Kakpo, 2013), en forêt inondable (Adjakpa et al., 2011; Hèdégbètan, 2011), en forêt dense sèche (Biaou, 1999; Yedomonhan et al., 2008 ; Woegan et al., 2013) et en formations saxicoles (Oumorou, 2003; Yedomonhan et al., 2008). Les travaux effectués par Kakpo et al. (2018) ont révélé que l'espèce a une structure spatiale agrégative dans un rayon de $10 \mathrm{~m}$ autour d'un pied repère avec une tendance à la dispersion entre $10 \mathrm{~m}$ et $30 \mathrm{~m}$ aussi bien en forêt dense humide semi-décidue qu'en forêt galerie. Sa présence est également signalée au Libéria (Odugbemi, 2006), en Centrafrique (Motte, 1980) et au Cameroun (Ndah et al., 2013) (Figure 3). Au Bénin, l'espèce est présente dans les districts phytogéographiques côtier, Pobè, Plateau, 
Vallée de l'Ouémé, Bassila, Zou et BorgouSud (Figure 4) (Adomou, 2005 ; Akoègninou et al., 2006 ; Dansi et al., 2010). Elle se trouve dans les zones guinéenne et soudanoguinéenne (Akoègninou et al., 2006 ; Achigan-Dako et al., 2010 ; Assogbadjo et al., 2012).

\section{PHENOLOGIE}

Au sud-ouest du Nigéria, la floraison de $C$. millenii a lieu de janvier à mars et la fructification se déroule de février à mai (Adegbola et Morakinyo, 2006). Au Bénin, les phases de floraison et fructification de l'espèce interviennent en février, mars, août, novembre et décembre (Akoègninou et al., 2006).

\section{APPELLATIONS UTILISATIONS ECONOMIQUE \\ LOCALES, ET IMPORTANCE}

Les noms locaux de $C$. millenii varient d'un groupe socioculturel à un autre (Tableau 1). L'usage de C. millenii dans l'alimentation est documenté par plusieurs auteurs (Tableau 2). Les jeunes feuilles de l'espèce sont faiblement consommées (Dansi et al., 2008). De même, l'utilisation de ses feuilles dans le traitement traditionnel de plusieurs maladies a été rapportée (Tableau 3) du fait de leurs propriétés médicinales (Itoandon et al., 2016). L'extrait de l'écorce contient des principes actifs pouvant être utilisés dans le contrôle de l'infestation des grains de maïs par Prostephanus truncatus (Osipitan et al., 2010). Son bois est également utilisé à diverses fins (Tableau 4). Au Nigéria, $C$. millenii est une espèce à valeur économique élevée (Bascom, 1951; Nyadanu et al., 2016). Elle fait également l'objet de commerce au Togo (Kokou et al., 2013) et au Bénin (Adomou et al., 2012).

\section{PROPRIETES CHIMIQUES}

$\mathrm{Au}$ plan chimique, plusieurs travaux (Tableaux 5 et 6 ) ont été réalisés sur $C$. millenii. La teneur en vitamine $\mathrm{C}$ de la plante est de $144,26 \pm 0,52 \mathrm{mg} / 100 \mathrm{~g}$ et de $953,33 \pm 0,78 \mathrm{mg} / 100 \mathrm{~g}$ respectivement pour la graine et le mésocarpe (Bello et al., 2008) et de 73,30 $\pm 3,54 \mathrm{mg} / \%$ pour la pulpe (Olayiwola et al., 2013). La graine, la pulpe, l'épicarpe, le mésocarpe, la feuille, l'écorce de la racine et l'écorce du tronc ont également fait l'objet d'études phytochimiques (Tableau 7). Les résultats du Criblage antioxidant et antimicrobien (Tableaux 8 et 9) effectué par Orisakeye et Odjo (2013) indiquent que les extraits bruts de diverses parties de $C$. millenii ont montré une réaction avec la méthode DPPH (2,2-diphenyl-1-dipicrylhydrazyl) après quelques minutes sauf la feuille. La graine a montré une réaction immédiate de couleur correspondant à une forte activité antioxydante. Les écorces de la racine et de la tige ont montré une réaction de couleur après 10 minutes signifiant une faible activité antioxydante. L'épicarpe possède une forte activité antioxydante après 5 minutes. Dans cette étude, différentes parties des extraits bruts de $C$. millenii ont été soumises au test préliminaire d'antibiotique. Les feuilles de $C$. millenii ont été actives contre Pseudomonas aeruginosa à un diamètre minimal de la zone d'inhibition de $6,5 \mathrm{~mm}$ et Bacillus subtilis à un diamètre de $6,5 \mathrm{~mm}$ mais inactives contre Escherichia coli et Staphylococus aureus. L'épicarpe n'a montré aucune activité avec $E$. coli mais a été actif contre $S$. aureus à un diamètre minimal de la zone d'inhibition de $15 \mathrm{~mm}, P$. aeruginosa à un diamètre de $6 \mathrm{~mm}$, B. subtilis à un diamètre de $10 \mathrm{~mm}$. La graine, l'écorce de la racine et du tronc n'ont montré aucune activité avec les organismes. De même, le criblage antimicrobien réalisé par Sonibare et al. (2009) indique que l'extrait des feuilles de $C$. millenii inhibe la croissance de $P$. aeruginosa à un diamètre minimal de zone d'inhibition de $8,0 \mathrm{~mm}$ avec une concentration minimale inhibitrice (CMI) de $90 \mathrm{mgml}^{-1}$. Aussi ont-ils souligné que cet extrait inhibe la croissance d'Aspergillus Niger dans une CMI de $90 \mathrm{mgml}^{-1}$ mais ne peut inhiber la croissance de Candida albicans qu'à des concentrations plus élevées de $120 \mathrm{mgml}^{-1}$ et $150 \mathrm{mgml}^{-1}$ (Tableau 10).

Les études menées sur les caractéristiques anatomiques du bois de $C$. millenii ont révélé que l'espèce dispose des cristaux monohidriques (Akinloye, 2012). 
L'évaluation de la toxicité des organes de $C$. millenii a permis d'obtenir une dose létale (D50) de $5000 \mathrm{mg} / \mathrm{kg}$ par voie orale et 2154 $\mathrm{mg} / \mathrm{kg}$ par voie intrapéritonéale pour la feuille (Oyemitan et al., 2016) et de 2645, $75 \mathrm{mg} / \mathrm{kg}$ par voie intrapéritonéale pour la graine (Ubon et al., 2017).

\section{PROPAGATION ET GENETIQUE}

C. millenii se régénère par graine (Adebola et Morakinyo, 2006). Sa dispersion est assurée par les primates (Ademoh et al., 2017). Le semis de la graine issue du croisement entre $C$. millenii et Cola nitida n'a abouti à aucun résultat (Morakinyo, 1995). De même, les résultats des études effectuées sur la propagation végétative (greffage) des espèces du genre Cola ont montré qu'il y a une incompatibilité entre $C$. millenii et Cola acuminata (Oladokun, 1986).

\section{INSECTES NUISIBLES, MENACES ANTHROPIQUES ET CONSERVATION}

Plusieurs insectes nuisibles des espèces du genre Cola ont été identifiés. Il s'agit essentiellement d'insectes de la famille des Isoptères, Coléoptères, Lépidoptères, Orthoptères, Hémiptères, Thysanoptères, et Diptères (Pujol, 1957). Plus particulièrement par rapport à $C$. millenii, les poux du Cacaoyer (Sahlbergella singularis et Distantiella theobromae) ont été identifiées comme des insectes responsables de lésions sur les tiges et follicules et de formation de chancres sur les fruits de C. millenii (Pujol, 1957). De même $C$. millenii est attaquée (inflorescences, rameaux, feuilles et bourgeons) par des cochenilles telles que Pseudococcus citri et Pseudococcus njalensis (ibid.). C. millenii subit des menaces dans son milieu naturel. Les principaux facteurs de menaces qui pèsent sur l'espèce au Ghana et en Côte d'Ivoire sont les défrichements à des fins agricoles (Anglaaere et al., 2011; Koulibaly et al., 2016). Cependant, elle bénéficie de protection dans certains systèmes agroforestiers traditionnels (Asase et Teteh, 2010; Assogbadjo et al., 2012; Jagoret et al., 2012; Nyadanu et al., 2016).

\section{GAP DE CONNAISSANCES ET PERSPECTIVES DE RECHERCHE}

C. millenii est une espèce à aire de distribution restreinte aussi bien en Afrique qu'au Bénin. Elle est rencontrée dans divers habitats (forêt dense humide semi-décidue, forêt dense sèche, forêt galerie, forêt marécageuse, forêt inondable et formations saxicoles). Connue sous plusieurs noms, elle est utilisée dans maints domaines. Bien que Akoègninou et al. (2006) aient précisé les périodes de floraison et fructification (février, mars, août, novembre et décembre) à l'échelle du Bénin, aucune étude n'a été faite à ce jour sur la phénologie de l'espèce dans ses différents phytodistricts de présence. Ces auteurs ont aussi indiqué que les mois mentionnés sont basés sur les exemplaires d'herbiers et que par conséquent ces dates ne sont pas nécessairement complètes. L'étude de la phénologie de l'espèce basée sur une démarche méthodologique rigoureuse s'avère donc nécessaire pour clarifier la situation afin de lever toute équivoque.

Plusieurs travaux ont été conduits sur la composition chimique de cette espèce. Selon Bello et al. (2008), on note la présence de sucre réducteur dans la graine de $C$. millenii alors que Adjayi et al. (2013) ont signalé l'absence de ce même constituant chimique dans le même organe de la plante. De même, la présence de tannin a été signalée dans la graine de la plante par plusieurs auteurs (Bello et al., 2008 ; Giwa, 2012 ; Ajayi et Ojelere, 2013 ; Borokini et al., 2014) alors que son absence y a été rapportée dans les travaux de Ubon et al. (2017). Il se trouve là une contradiction qui mérite d'être élucidée. Cependant, cet état de chose peut s'expliquer par la qualité du solvant utilisé ou du type de sol sur lequel se trouve l'espèce (Lalèyè et al., 2015). La propriété antimicrobienne de la feuille justifie son utilisation dans le traitement des maladies comme : la teigne, la gale, la dysenterie, la gonorrhée et la conjonctivite.

Concernant l'importance médicinale de la plante, seul l'usage des feuilles a été rapporté. Les constituants de la plante en vitamine ne sont pas suffisamment évalués. 
C'est seulement au Nigéria que la composition de la pulpe, de la graine et du mésocarpe de l'espèce en vitamine $\mathrm{C}$ a été rapportée. La teneur de la feuille en vitamine est à évaluer. Sur le plan économique, Bien que Nyadanu et al. (2016) aient indiqué qu'au Nigéria $C$. millenii est une espèce à valeur économique élevée, aucune information n'est disponible sur le revenu généré par cette ressource. Son importance économique n'est pas encore bien évaluée.

Par ailleurs, il n'y a quasiment pas d'informations sur l'étude génétique et la sylviculture de l'espèce. Par rapport à sa propagation, en se référant aux études effectuées par Yakubu et al. (2014) sur Garcinia kola, on peut envisager des essais sur le greffage et le bourgeonnement de l'espèce.

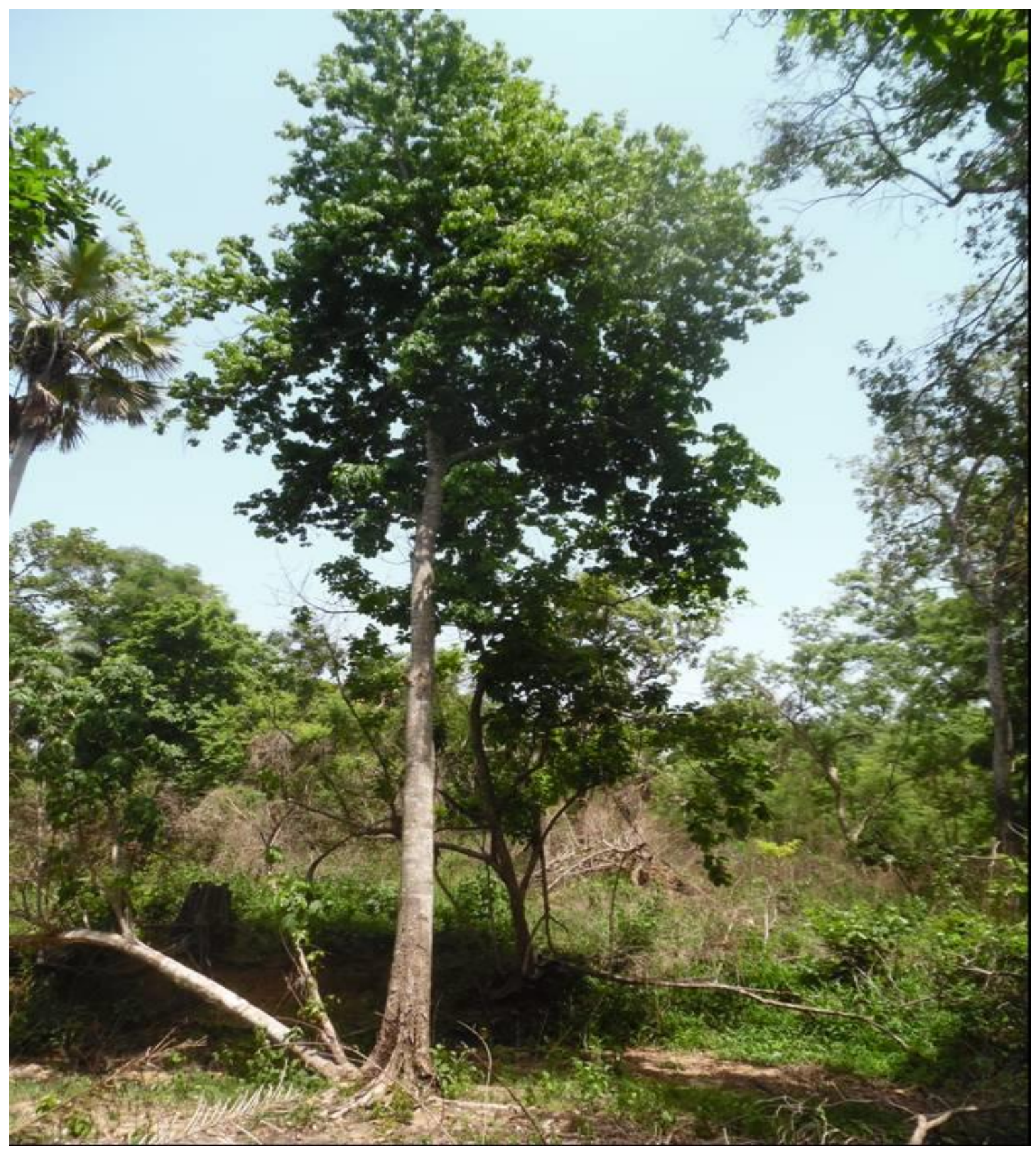

Figure 1: Arbre de C. millenii. (Source : Lawin, 2016). 


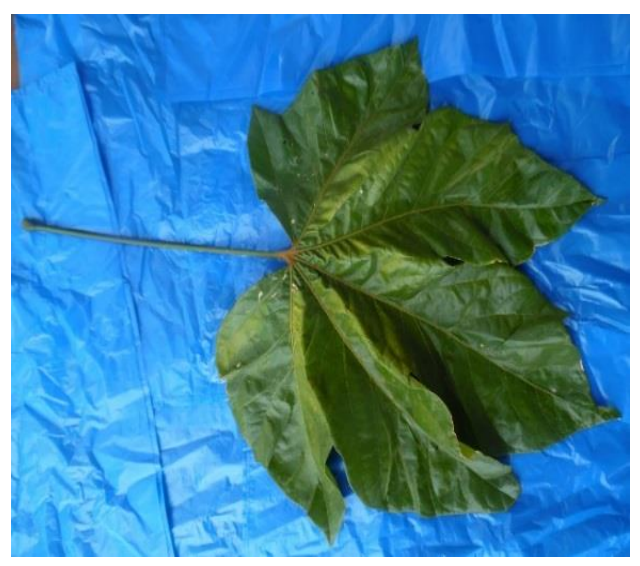

a

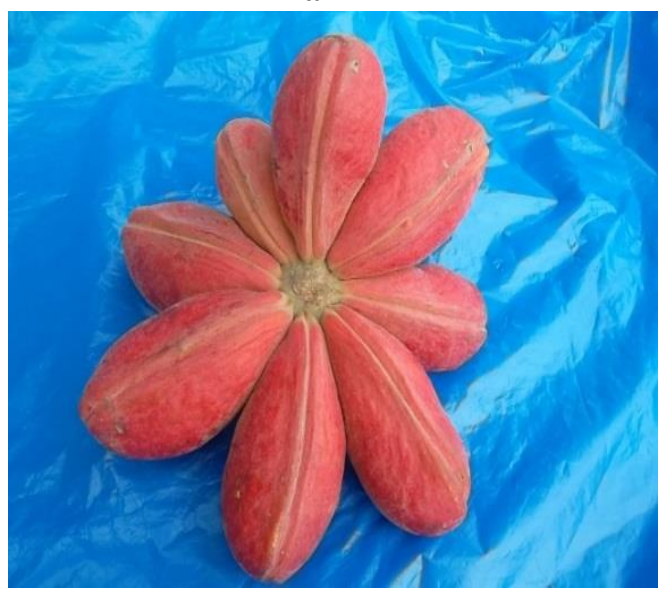

c

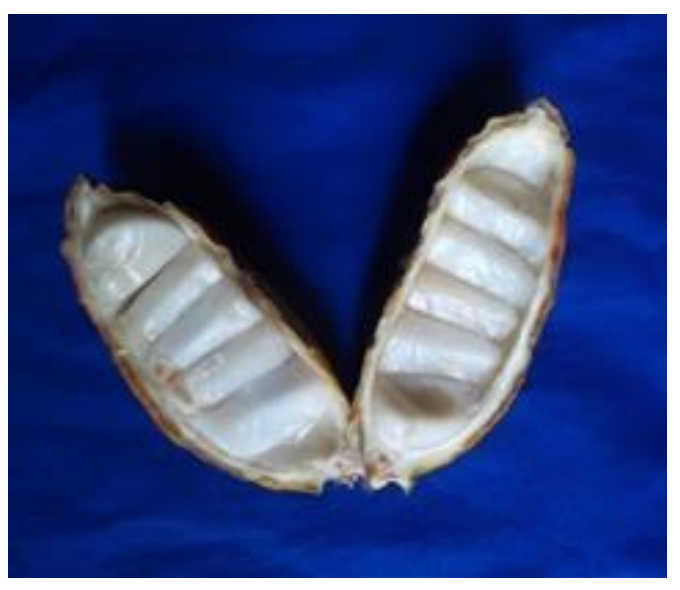

e

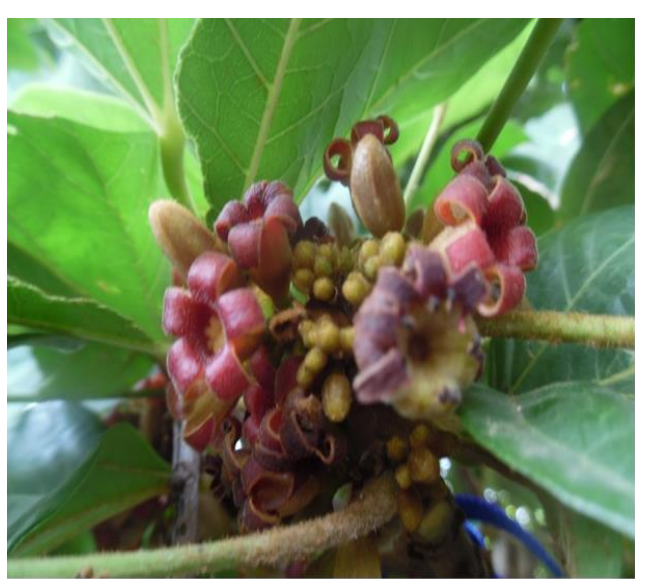

b

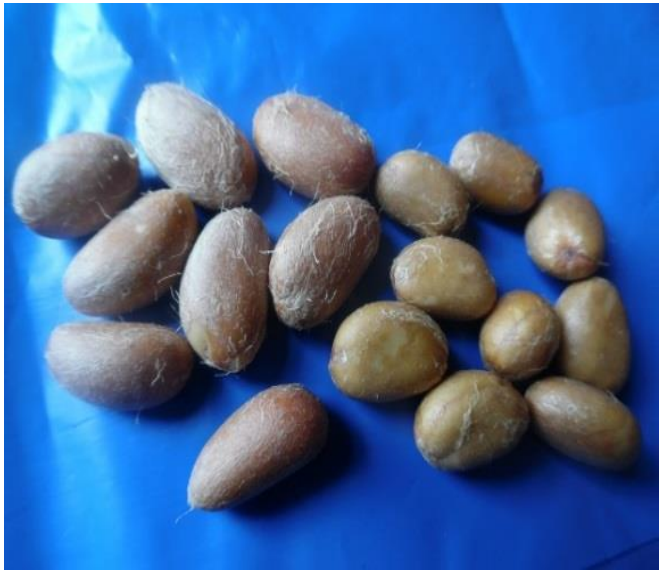

$\mathrm{d}$

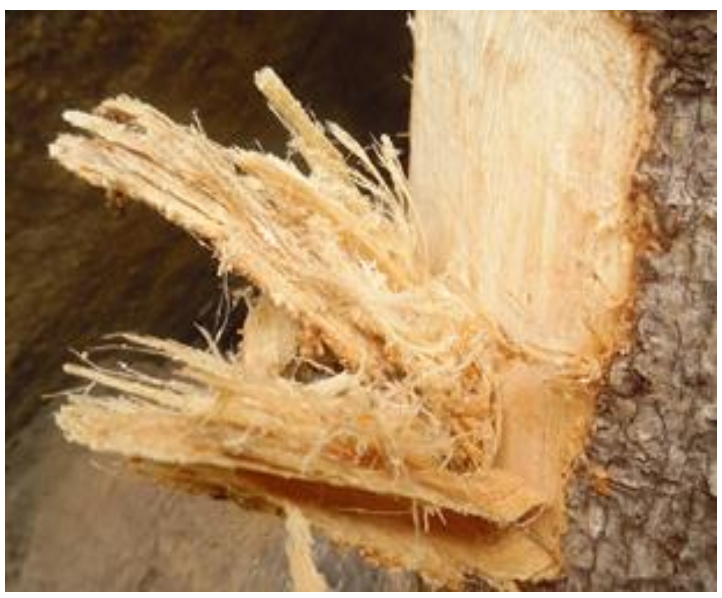

f

Figure 2: Différents organes de C. millenii (Feuille (a), Fleurs (b), Fruit (c), graine (d), Fruit ouvert montrant les graines enfouies dans la pulpe (e), Ecorce (f)). (source : Lawin, 2016). 


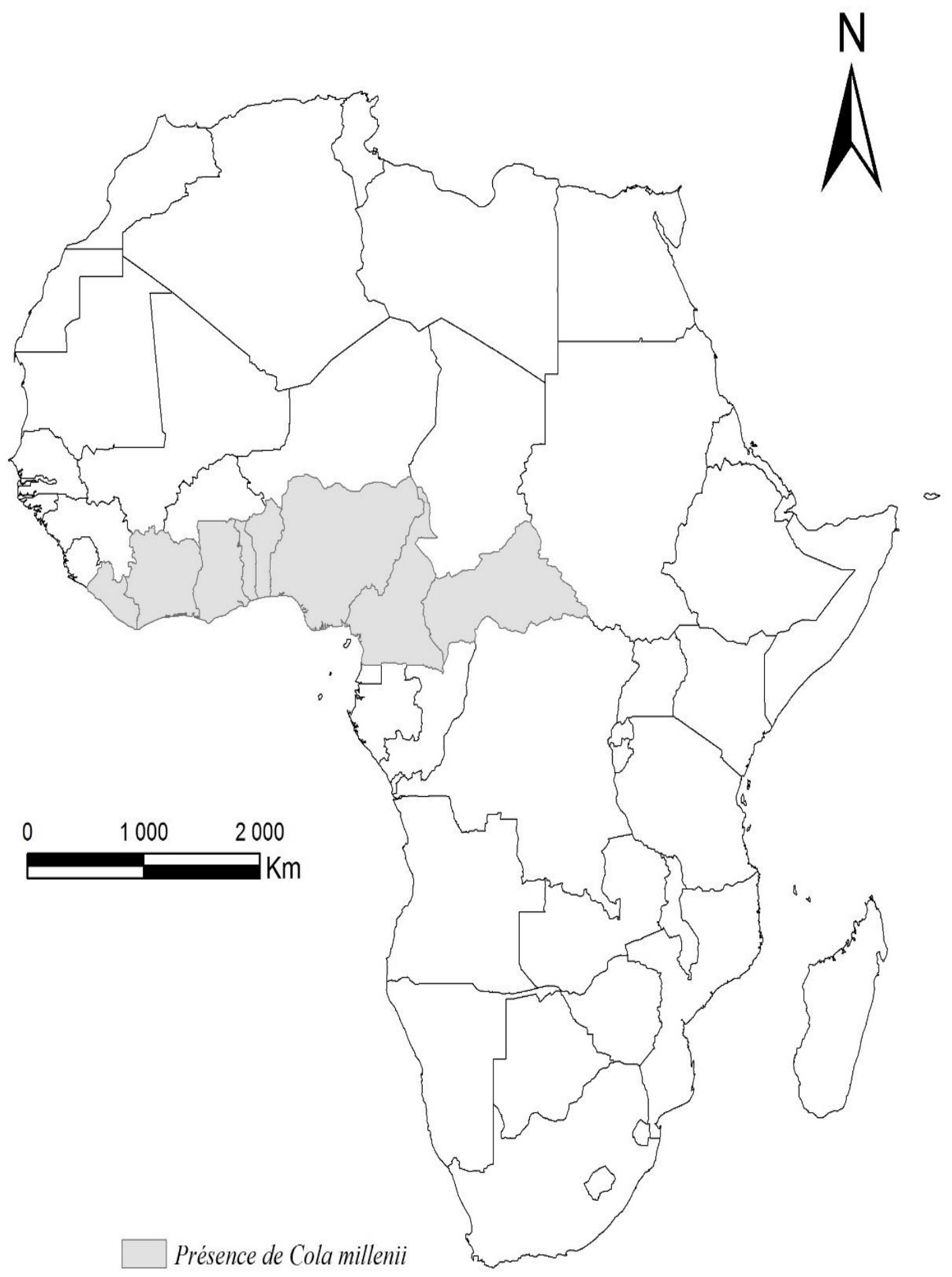

Figure 3: Distribution naturelle de C. millenii en Afrique. (Source : Lawin, 2017). 


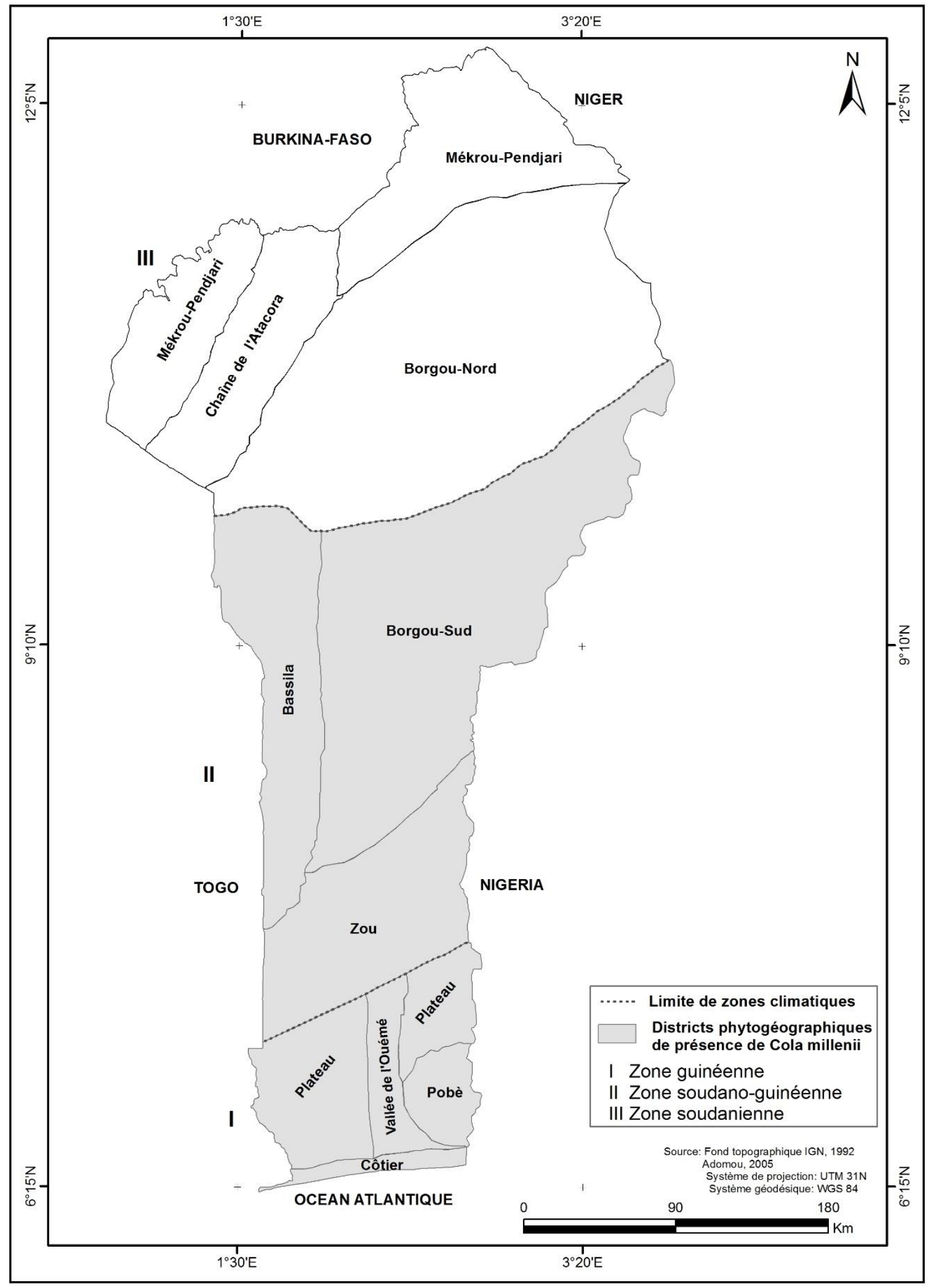

Figure 4: Distribution naturelle de C. millenii au Bénin. (Source : Lawin, 2017). 
Tableau 1 : Noms communs de $C$. millenii.

\begin{tabular}{|c|c|c|c|c|}
\hline $\mathbf{N}^{\circ}$ & Langue & Noms communs & Pays & Références \\
\hline 1 & Français & Kola du singe & - & $\begin{array}{l}\text { Akoègninou et al., } 2006 \text {; de Souza, } \\
2008\end{array}$ \\
\hline 2 & Anglais & Monkey Kola & Nigéria & $\begin{array}{l}\text { Orisakeye et Ojo, 2013; Ajayi et } \\
\text { Ojelere, 2013; Ubon et al. (2017); } \\
\text { Alaribe et Coker, } 2014\end{array}$ \\
\hline 3 & Fon & $\begin{array}{l}\text { Aloviatoon, Aze loko, Bambo, } \\
\text { Pafoli, Afutukolo }\end{array}$ & Bénin & de Souza, 2008 \\
\hline 4 & $\begin{array}{l}\text { Yoruba, } \\
\text { Nagot }\end{array}$ & $\begin{array}{l}\text { Kimelakao, Gbototo, Aluganle, } \\
\text { Akabame }\end{array}$ & Bénin & de Souza, 2008 \\
\hline 5 & Yoruba & $\begin{array}{l}\text { Atewo-edun } \\
\text { Obi-edun }\end{array}$ & Nigéria & $\begin{array}{l}\text { Orisakeye et Ojo, } 2013 \\
\text { Adebola et Morakinyo, } 2006 \text {; Ajayi et } \\
\text { Ojelere, } 2013 \text {; Oyemitan et al., } 2016 \text {; } \\
\text { Ubon et al. (2017) }\end{array}$ \\
\hline 6 & $\begin{array}{l}\text { Nagot (de } \\
\text { Idadjo) }\end{array}$ & Igi allassa & Bénin & de Souza, 2008 ; Ceperley et al., 2010 \\
\hline 7 & Adja & Wawa & Bénin & de Souza, 2008 \\
\hline 8 & Sahouè & Dindonvema & Bénin & de Souza, 2008 \\
\hline 9 & Wachi & Sakpatati, Sokpekpeti & Bénin & $\begin{array}{l}\text { Adjanohoun et al., } 1989 \text {; de Souza, } \\
2008\end{array}$ \\
\hline 10 & Aïzo & Aziokèkè & Bénin & Achigan-Dako et al., 2009 \\
\hline 11 & $\begin{array}{l}\text { Ewe, } \\
\text { Mina }\end{array}$ & Assiviatoe, Kpando & Togo & $\begin{array}{l}\text { Denou et al., } 2016 \text {; Koudouvo et al., } \\
\text { 2016; } \\
\text { Kpodar et al., } 2016\end{array}$ \\
\hline 12 & Bété & Bô ; Bouti & $\begin{array}{l}\text { Côte } \\
\text { d'Ivoire }\end{array}$ & N'dri et al., 2008 \\
\hline 13 & Ewondo & Efok & Cameroun & Nkongmeneck, 1982 \\
\hline 14 & Ibo & achi okokoro & Nigéria & Orisakeye et Ojo, 2013 \\
\hline 15 & Ibibio & Mba utong-ita & Nigéria & Ubon et al. (2017) \\
\hline 16 & - & Ananse adodowa & Ghana & Ubon et al. (2017) \\
\hline
\end{tabular}

Tableau 2 : Usage alimentaire de C. millenii.

\begin{tabular}{lll}
\hline Partie consommée & Mode de consommation & Références \\
\hline Pulpe & Cru & Atato et al., 2011; Codjia et al., 2003; \\
& & $\begin{array}{l}\text { Assogbadjo et al., 2012; Nyadanu et al., } \\
2016\end{array}$ \\
\hline Graine & - & Atato et al., 2010 \\
\hline Jeune feuille & Légume feuille (sauce) & Achigan-Dako et al., 2009; Dansi et al., \\
& & $2008 ;$ N'dri et al., 2008; Amoussou, 2005 \\
\hline$-=$ non précisé & &
\end{tabular}


Tableau 3 : Usage médicinal de $C$. millenii.

\begin{tabular}{|c|c|c|c|}
\hline $\begin{array}{l}\text { Organe } \\
\text { utilisé }\end{array}$ & Mode de préparation & Maladies traitées & Références \\
\hline \multirow[t]{10}{*}{ Feuille } & Décoction & Paludisme & $\begin{array}{l}\text { Denou et al., 2016; Koudouvo et al., } \\
\text { 2016; Adomou et al., 2012; Amontcha } \\
\text { et al., 2011; Amoussou, 2005; Hermans } \\
\text { et al., } 2004\end{array}$ \\
\hline & Infusion & Fièvre & Amoussou, 2005 \\
\hline & - & Ictère & $\begin{array}{l}\text { Assogbadjo et al., } 2011 \text {; Akoègninou et } \\
\text { al., } 2006 \text {; Amoussou, } 2005\end{array}$ \\
\hline & - & $\begin{array}{lr}\begin{array}{lr}\text { Teigne, } & \text { gale, } \\
\text { dysenterie } & \text { et } \\
\text { conjonctivite } & \end{array}\end{array}$ & $\begin{array}{l}\text { Odugbemi, 2006; Alaribe et Coker, } \\
2014\end{array}$ \\
\hline & Mixture (en mélange) & Gonorrhée & Ndah et al., 2012 ; Odugbemi, 2006 \\
\hline & Décoction ou macération & $\begin{array}{l}\text { Abcès et éruptions } \\
\text { cutanées }\end{array}$ & $\begin{array}{l}\text { Akoègninou et al., } 2006 \text {; Adjanohoun } \\
\text { et al., } 1989 \text {; Adeogun et al., } 2014\end{array}$ \\
\hline & $\begin{array}{l}\text { Trituration } \\
\text { kilogramme de feuilles } \\
\text { dans deux litres d'eau; } \\
\text { filtrer et ajouter } 54 \\
\text { morceaux de sucre }\end{array}$ & Cardiopathie & Adjanohoun et al., 1989 \\
\hline & Décoction & Stérilité féminine & Houmenou et al., 2017 \\
\hline & Décoction & $\begin{array}{l}\text { Affections } \\
\text { hépathiques }\end{array}$ & Sangaré et al., 2012; Kpodar et al., 2016 \\
\hline & - & Salmonellose & Dougnon et al., 2017 \\
\hline- & - & Varicelle & Adjanohoun et al., 1989 \\
\hline
\end{tabular}

Tableau 4: Usages du bois de C. millenii.

\begin{tabular}{lll}
\hline Organe utilisé & Utilisations & Références
\end{tabular}

Bois Construction des maisons et fabrication des pirogues Ceperley et al., 2010 par les populations de Idadjo (Bénin)

Fabrication de l'arbalète au Nigeria ; Fabrication des Odugbemi, 2006 pièges à rats et des arcs au Libéria

Utiliser comme outils de capture des insectes par les Pascual-Garrido et al., 2011 chimpanzés au Nigéria

Bois de feu et de service (Bénin) Assogbadjo et al., 2011

Bois énergie et bois d'œuvre (Bénin) Dossou et al. , 2012 
Tableau 5: Composition en divers éléments nutritifs des organes de $C$. millenii.

\begin{tabular}{|c|c|c|c|c|c|c|c|c|c|c|}
\hline $\begin{array}{l}\text { Organes } \\
\text { de } C \text {. } \\
\text { millenii }\end{array}$ & Humidité & Lipide brut & Protéine brut & Cendre & Fibre brut & Glucide & $\begin{array}{l}\text { Sucre } \\
\text { réducteur }\end{array}$ & $\begin{array}{l}\text { Sucres } \\
\text { totaux }\end{array}$ & $\begin{array}{l}\text { Valeur } \\
\text { énergétique } \\
\text { (kcal/100kg) }\end{array}$ & Références \\
\hline & $19,00 \pm 0,000 \%$ & $8,72 \pm 0.010 \%$ & $12,52 \pm 0,400 \%$ & $8,31 \pm 0,012 \%$ & $5,28 \pm 0,020 \%$ & $51,54 \pm 0,018 \%$ & - & - & 1411,66 & $\begin{array}{l}\text { Ajayi et } \\
\text { Ojelere, } 2013\end{array}$ \\
\hline & $\begin{array}{l}16,65 \pm 0,43 \\
g / 100 g\end{array}$ & $\begin{array}{l}43,83 \pm 0,01 \\
g / 100 g\end{array}$ & $\begin{array}{l}5,78 \pm 0,06 \\
g / 100 \mathrm{~g}\end{array}$ & $\begin{array}{l}2,76 \pm 0,49 \\
\mathrm{~g} / 100 \mathrm{~g}\end{array}$ & $\begin{array}{l}2,23 \pm 0,65 \\
\mathrm{~g} / 100 \mathrm{~g}\end{array}$ & $\begin{array}{l}28,73 \pm 0,15 \\
g / 100 g\end{array}$ & - & - & - & $\begin{array}{l}\text { Borokini et al., } \\
2014\end{array}$ \\
\hline Graine & $\begin{array}{l}10.00 \pm 0,01 \\
\mathrm{~g} / 100 \mathrm{~g}\end{array}$ & $\begin{array}{l}40,0 \pm 1,16 \\
g / 100 \mathrm{~g}\end{array}$ & $\begin{array}{l}9,19 \pm 0,62 \\
g / 100 \mathrm{~g}\end{array}$ & $\begin{array}{l}3,00 \pm 1,41 \\
\mathrm{~g} / 100 \mathrm{~g}\end{array}$ & $\begin{array}{l}4,03 \pm 0,01 \\
\mathrm{~g} / 100 \mathrm{~g}\end{array}$ & - & $\begin{array}{l}14,67 \pm 0,18 \\
\mathrm{mg} / \mathrm{g}\end{array}$ & $\begin{array}{l}54,63 \pm 0,01 \\
\mathrm{mg} / \mathrm{g}\end{array}$ & - & $\begin{array}{l}\text { Bello et al., } \\
2008\end{array}$ \\
\hline Pulpe & $\begin{array}{l}18,95 \pm 0,33 \\
g / 100 g\end{array}$ & $\begin{array}{l}30,03 \pm 1,00 \\
g / 100 \mathrm{~g}\end{array}$ & $\begin{array}{l}4,40 \pm 0,43 \\
g / 100 \mathrm{~g}\end{array}$ & $\begin{array}{l}3,15 \pm 0,25 \\
\mathrm{~g} / 100 \mathrm{~g}\end{array}$ & $\begin{array}{l}12,83 \pm 0,71 \\
\mathrm{~g} / 100 \mathrm{~g}\end{array}$ & $\begin{array}{l}30,30 \pm 0,93 \\
\mathrm{~g} / 100 \mathrm{~g}\end{array}$ & - & - & - & $\begin{array}{l}\text { Borokini et al., } \\
2014\end{array}$ \\
\hline Mésocarpe & $\begin{array}{l}19,0 \pm 1,41 \\
\mathrm{~g} / 100 \mathrm{~g}\end{array}$ & $\begin{array}{l}37,0 \pm 1,41 \\
\mathrm{~g} / 100 \mathrm{~g}\end{array}$ & $\begin{array}{l}7,44 \pm 0,01 \\
\mathrm{~g} / 100 \mathrm{~g}\end{array}$ & $\begin{array}{l}2.00 \pm 0,01 \\
\mathrm{~g} / 100 \mathrm{~g}\end{array}$ & $\begin{array}{l}10,0 \pm 0,62 \\
\mathrm{~g} / 100 \mathrm{~g}\end{array}$ & $\begin{array}{l}34,56 \pm 0,62 \\
\mathrm{~g} / 100 \mathrm{~g}\end{array}$ & $\begin{array}{l}4.63 \pm 0.26 \\
\mathrm{mg} / \mathrm{g}\end{array}$ & $\begin{array}{l}47.08 \pm 1.22 \\
\mathrm{mg} / \mathrm{g}\end{array}$ & - & $\begin{array}{l}\text { Bello et al., } \\
2008\end{array}$ \\
\hline *Feuille & - & $0,1 \pm 0,1 \%$ & $10,1 \pm 1,0 \%$ & $10,2 \pm 2,0 \%$ & $18,4 \pm 3,5 \%$ & - & - & $\begin{array}{l}54,7 \pm 2,0 \\
\%\end{array}$ & - & $\begin{array}{l}\text { Amoussou, } \\
2005\end{array}$ \\
\hline
\end{tabular}

*\% matière sèche pour $100 \mathrm{~g} ;-$ = pas d'informations

Tableau 6: Composition en éléments minéraux des organes de C. millenii.

\begin{tabular}{|c|c|c|c|c|c|c|c|c|c|c|c|}
\hline $\begin{array}{l}\text { Organes de } \\
\text { C. millenii }\end{array}$ & $\mathbf{C a}$ & Mg & & $\mathbf{K}$ & $\mathbf{N a}$ & Mn & $\mathbf{F e}$ & Phosphore & Zn & $\mathrm{Cu}$ & Références \\
\hline \multirow[t]{2}{*}{ Pulpe } & $\begin{array}{l}394,78 \\
\mathrm{mg} / 100 \mathrm{~g}\end{array}$ & $\begin{array}{l}610,29 \\
\mathrm{mg} / 100 \mathrm{~g}\end{array}$ & & $\begin{array}{l}356,47 \\
\mathrm{mg} / 100 \mathrm{~g}\end{array}$ & $\begin{array}{l}187,15 \\
\mathrm{mg} / 100 \mathrm{~g}\end{array}$ & $\begin{array}{l}555,23 \\
\mathrm{mg} / 100 \mathrm{~g}\end{array}$ & $\begin{array}{l}1901,75 \\
\mathrm{mg} / 100 \mathrm{~g}\end{array}$ & - & $\begin{array}{l}481,53 \\
\mathrm{mg} / 100 \mathrm{~g}\end{array}$ & $\begin{array}{l}7,80 \\
\mathrm{mg} / 100 \mathrm{~g}\end{array}$ & Borokini et al., 2014 \\
\hline & $\begin{array}{l}87,17 \pm \\
11,18\end{array}$ & $\begin{array}{l}68,16 \pm \\
\mathrm{mg} / 100 \mathrm{~g}\end{array}$ & 4,45 & $\begin{array}{l}1103,46 \pm \\
9.38\end{array}$ & $\begin{array}{l}11,22 \pm \\
0,21\end{array}$ & $\begin{array}{l}40,52 \pm 4,63 \\
\mathrm{mg} / 100 \mathrm{~g}\end{array}$ & $\begin{array}{l}0,85 \pm 0,03 \\
\mathrm{mg} / 100 \mathrm{~g}\end{array}$ & $\begin{array}{l}42,53 \pm 2,91 \\
\mathrm{mg} / 100 \mathrm{~g}\end{array}$ & $\begin{array}{l}0,26 \pm 0.03 \\
\mathrm{mg} / 100 \mathrm{~g}\end{array}$ & $\begin{array}{l}0,00 \\
\mathrm{mg} / 100 \mathrm{~g}\end{array}$ & Olayiwola et al., 2013 \\
\hline
\end{tabular}


I. F. LAWIN et al. / Int. J. Biol. Chem. Sci. 12(3): 1494-1515, 2018

\begin{tabular}{|c|c|c|c|c|c|c|c|c|c|c|}
\hline & $\mathrm{mg} / 100 \mathrm{~g}$ & & $\mathrm{mg} / 100 \mathrm{~g}$ & $\mathrm{mg} / 100 \mathrm{~g}$ & & & & & & \\
\hline \multirow[t]{3}{*}{ Graine } & $\begin{array}{l}168,73 \\
\mathrm{mg} / 100 \mathrm{~g}\end{array}$ & $\begin{array}{l}459,47 \\
\mathrm{mg} / 100 \mathrm{~g}\end{array}$ & $\begin{array}{l}417,72 \\
\mathrm{mg} / 100 \mathrm{~g}\end{array}$ & $\begin{array}{l}140,99 \\
\mathrm{mg} / 100 \mathrm{~g}\end{array}$ & $\begin{array}{l}100,02 \\
\mathrm{mg} / 100 \mathrm{~g}\end{array}$ & $\begin{array}{l}446,56 \\
\mathrm{mg} / 100 \mathrm{~g}\end{array}$ & - & $\begin{array}{l}310,26 \\
\mathrm{mg} / 100 \mathrm{~g}\end{array}$ & $\begin{array}{l}9,90 \\
\mathrm{mg} / 100 \mathrm{~g}\end{array}$ & Borokini et al., 2014 \\
\hline & $\begin{array}{l}23,8 \pm 3,50 \\
\mathrm{mg} / \mathrm{L}\end{array}$ & $2,16 \pm 0,09 \mathrm{mg} / \mathrm{L}$ & $\begin{array}{l}4,34 \pm 0,35 \\
\mathrm{mg} / \mathrm{L}\end{array}$ & $\begin{array}{l}4,80 \pm 0,14 \\
\mathrm{mg} / \mathrm{L}\end{array}$ & $\begin{array}{l}0,50 \pm 0,04 \\
\mathrm{mg} / \mathrm{L}\end{array}$ & $\begin{array}{l}0,36 \pm 0,09 \\
\mathrm{mg} / \mathrm{L}\end{array}$ & - & $\begin{array}{l}3,05 \pm 0,50 \\
\mathrm{mg} / \mathrm{L}\end{array}$ & - & Ajayi et Ojelere, 2013 \\
\hline & $\begin{array}{l}1681,35 \pm \\
30,76 \\
(\mathrm{mg} / \mathrm{kg})\end{array}$ & $\begin{array}{l}3934,01 \pm 87,19 \\
(\mathrm{mg} / \mathrm{kg})\end{array}$ & $\begin{array}{l}4776,90 \pm \\
18,24 \\
(\mathrm{mg} / \mathrm{kg})\end{array}$ & $\begin{array}{l}1387,90 \pm \\
0,99 \\
(\mathrm{mg} / \mathrm{kg})\end{array}$ & $\begin{array}{ll}100,20 \quad \pm \\
0,28 \\
(\mathrm{mg} / \mathrm{kg})\end{array}$ & $\begin{array}{ll}481,3 & \pm \\
1,41 & \\
(\mathrm{mg} / \mathrm{kg}) & \end{array}$ & - & $\begin{array}{l}319,05 \\
\pm 0,35 \\
(\mathrm{mg} / \mathrm{kg})\end{array}$ & $\begin{array}{l}96,53 \quad \pm \\
0,66 \\
(\mathrm{mg} / \mathrm{kg})\end{array}$ & \multirow{2}{*}{ Bello et al., (2008) } \\
\hline Mésocarpe & $\begin{array}{l}11696,92 \\
\pm \quad 28,3 \\
(\mathrm{mg} / \mathrm{kg})\end{array}$ & $\begin{array}{l}6916,7 \pm 19,38 \\
(\mathrm{mg} / \mathrm{kg})\end{array}$ & $\begin{array}{l}3458,95 \pm \\
21,76 \\
(\mathrm{mg} / \mathrm{kg})\end{array}$ & $\begin{array}{l}1834,16 \pm \\
28,25 \\
(\mathrm{mg} / \mathrm{kg})\end{array}$ & $\begin{array}{l}973,48 \\
\pm 60,26 \\
(\mathrm{mg} / \mathrm{kg})\end{array}$ & $\begin{array}{l}1971,29 \pm 3 \\
5,09 \\
(\mathrm{mg} / \mathrm{kg})\end{array}$ & - & $\begin{array}{l}559,74 \quad \pm \\
34,22 \\
(\mathrm{mg} / \mathrm{kg})\end{array}$ & $\begin{array}{l}65,71 \quad \pm \\
3,46 \\
(\mathrm{mg} / \mathrm{kg})\end{array}$ & \\
\hline Feuille & $\begin{array}{l}791,0 \pm 0,7 \\
\mathrm{mg} / 100 \mathrm{~g}\end{array}$ & - & - & - & - & $\begin{array}{l}19,0 \pm 0,1 \\
\mathrm{mg} / 100 \mathrm{~g}\end{array}$ & $\begin{array}{l}83,0 \pm 0,7 \\
\mathrm{mg} / 100 \mathrm{~g}\end{array}$ & - & - & Amoussou, 2005 \\
\hline
\end{tabular}

$-=$ pas d'informations

Tableau 7: Eléments phytochimiques des organes de C. millenii.

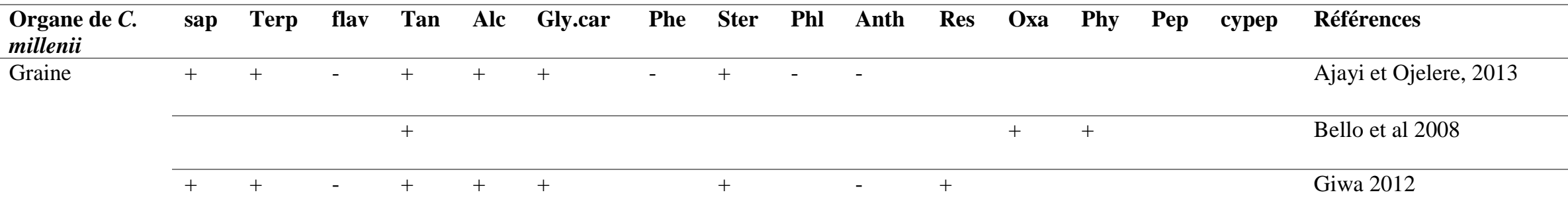


I. F. LAWIN et al. / Int. J. Biol. Chem. Sci. 12(3): 1494-1515, 2018

\begin{tabular}{|c|c|c|c|c|c|c|c|c|c|c|c|}
\hline & + & + & - & - & + & + & & - & & & Ubon et al, 2017 \\
\hline & & & & + & & & & & + & + & Borokini et al, 2014 \\
\hline \multirow[t]{2}{*}{ Pulpe } & + & + & - & + & + & + & + & - & + & & Giwa, 2012 \\
\hline & & & & + & & & & & + & + & Borokini et al, 2014 \\
\hline Epicarpe & & & & & & & + & & & + & OrisaKeye et ojo, 2013 \\
\hline Mésocarpe & & & & + & & & & & + & + & Bello et al 2008 \\
\hline Feuille & + & & & + & + & & & & & & Sonibare et al, 2009 \\
\hline $\begin{array}{l}\text { Ecorce de la } \\
\text { racine }\end{array}$ & & & & & & & + & & & & Orisakeye et ojo, 2013 \\
\hline \multirow[t]{2}{*}{ Ecorce du tronc } & & & & & + & & & & & & Adegoke et al, 1968 \\
\hline & & & & & & & & & & & Orisakeye et ojo, 2013 \\
\hline
\end{tabular}

Sap $=$ Saponine $;$ Terp $=$ terpène $;$ Flav $=$ flavonoïde $;$ Tan $=$ Tannin $;$ Alc $=$ Alcaloïde $;$

Gly. car $=$ Glycoside cardiaque $;$ Phe $=$ Phénol $;$ Ster $=$ Stéroïdes $;$ Phl $=$ phlobatannin $;$ Anth $=$ Anthraquinones $;$

Rés $=$ Résine $;$ Oxa $=$ Oxalate (sel de l'acide oxalique) $;$ Phy = Phytate (sel de l'acide phytique) Pep = Peptide $;$ Cypep = Cyclopeptide

$+=$ Présent ; - = Absent ; Par rapport aux cases vides, il n'y a pas d'information

Tableau 8: Activités antioxydantes des extraits bruts de différents organes de C. millenii.

\begin{tabular}{|c|c|c|}
\hline Organes & Temps mis pour l'apparition de la couleur (méthode DPPH) & Activité antioxydante \\
\hline Feuille & Pas de réaction & - \\
\hline Graine & Réaction immediate & Forte \\
\hline Ecorce de la racine & $10 \mathrm{~min}$ & Faible \\
\hline Ecorce de la tige & $10 \mathrm{~min}$ & Faible \\
\hline Epicarpe & $5 \mathrm{~min}$ & Forte \\
\hline
\end{tabular}

Source : Orisakeye et Odjo (2013) 
I. F. LAWIN et al. / Int. J. Biol. Chem. Sci. 12(3): 1494-1515, 2018

Tableau 9: Activités antimicrobiennes des extraits bruts d'organes de C. millenii en concentration de $80 \mathrm{mg} / \mathrm{ml}$ et en méthanol H20 (1 :1).

\begin{tabular}{|c|c|c|c|c|c|c|}
\hline \multirow[t]{2}{*}{ Organisme testé } & \multicolumn{6}{|c|}{ Diamètre de zone d'inhibition (mm) } \\
\hline & Feuille & Epicarpe & Graine & Ecorce de la racine & Ecorce du tronc & Solvant \\
\hline Escherichia coli & - & - & - & - & - & - \\
\hline Staphylococcus aureus & - & 15 & - & - & - & - \\
\hline Pseudomonas aeruginosa & 6,5 & 6 & - & - & - & - \\
\hline Bacilus subtilis & 6,5 & 10 & - & - & - & - \\
\hline
\end{tabular}

- = pas d'activité

Source : Orisakeye et Odjo (2013)

Tableau 10: Activités antimicrobiennes des extraits bruts des feuilles de $C$. millenii.

\begin{tabular}{|c|c|c|c|c|c|c|c|}
\hline \multirow{2}{*}{$\begin{array}{l}\text { Concentration Minimale } \\
\text { Inhibitrice (CMI) en } \\
\text { mgml }^{-1}\end{array}$} & \multicolumn{7}{|c|}{ Diamètre de zone d'inhibition (mm) } \\
\hline & Staphylococcus albus & S. aureus & B. subtilis & Klebsiella pneumoniae & P. aeruginosa & C. albicans & A. niger \\
\hline 10 & - & - & - & - & - & - & - \\
\hline 30 & - & - & - & - & - & - & - \\
\hline 60 & - & - & - & - & - & - & - \\
\hline 90 & - & - & - & - & $8.0 \pm 0.0$ & - & $8.6 \pm 1.1$ \\
\hline 120 & - & - & - & - & $12.0 \pm 1.0$ & $11.5 \pm 0.0$ & $11.3 \pm 0.03$ \\
\hline 150 & - & - & - & - & $18.0 \pm 0.0$ & $15.0 \pm 0.0$ & $13.6 \pm 1.1$ \\
\hline
\end{tabular}

- : absence d'activité antimicrobienne

Source : Sonibare et al. (2009). 


\section{Conclusion}

Cette synthèse bibliographique a permis de faire un bilan des travaux déjà réalisés sur $C$. millenii en Afrique en général et au Bénin en particulier. La taxonomie, la description botanique, la caractérisation chimique des différents organes de l'espèce sont bien fournies. Cependant, certains aspects restent sous documentés. Des travaux complémentaires méritent d'être entrepris sur la variation des usages, l'écologie, la morphologie, la caractérisation biochimique et la phénologie de l'espèce. Des études scientifiques futures devraient également se pencher sur sa valeur économique, sa sylviculture et sa diversité génétique en vue de sa meilleure connaissance, sa valorisation et sa conservation.

\section{CONFLIT D'INTERETS}

Les auteurs déclarent qu'il n'existe aucun conflit d'intérêts relatif à cet article.

\section{CONTRIBUTIONS DES AUTEURS}

IFL et $\mathrm{ABF}$ ont conçu l'idée du manuscrit, collecté et synthétisé les données bibliographiques. IFL est l'investigateur principal qui a rédigé le manuscrit; $\mathrm{ABF}, \mathrm{KG}$ et AEA ont lu le manuscrit; CAINO a supervisé les travaux et lu la version finale du manuscrit.

\section{REMERCIEMENTS}

Les auteurs remercient Dr. Kolawolé Valère SALAKO, Monsieur Onodjè Pierre AGBANI pour la relecture d'une version antérieure du manuscrit et la Fondation Internationale pour la Science pour avoir accordé une bourse (Grant $\left.n^{\circ} \mathrm{D} / 5875-1\right)$ à Iboukoun Fidèle LAWIN dans le cadre de ce travail. Adandé Belarmain Fandohan a été soutenu par le Return Fellowship de la Foundation Alexander von Humboldt.

\section{REFERENCES}

Achigan-Dako EG, Pasquini MW, Assogba-Komlan F, N'danikou S, Yédomonhan H, Dansi A, Ambrose-Oji B. 2010. Traditional Vegetables in Benin. Institut National des Recherches
Agricoles du Bénin. Imprimeries du CENAP: Cotonou.

Adebola PO, Morakinyo JA. 2006. Evaluation of morpho-agronomic variability of wild and cultivated kola (Cola species Schott et Endel.) in South Western Nigeria. Genetic Resources and Crop Evolution, 53: 687-694. DOI: http://doi.org/10.1007/s10722-004-35581

Adegoke E, Akinsanya A, Nagu A. 1968. Studies of Nigerian Medicinal Plants. A preliminary survey of Plant Alkaloids. Journal of the West African Science Association, 13:13-39.

Adekunle VAJ, Olagoke AO. 2008. Diversity and biovolume of tree species in natural forest ecosystem in the bitumenproducing area of ondo state, Nigeria: a baseline study. Biodivers Conserv., 17: 2735-2755. DOI: 10.1007/s10531-0079279-y

Ademoh FO, Muoghalu JI, Onwumere B. 2017. Temporal pattern of tree community dynamics in a secondary forest in southwestern Nigeria, 29 years after a ground fire. Global Ecology and Conservation, 9: 148-170. DOI: http://dx.doi.org/10.1016/j.gecco.2016.1 1.005

Adeniyi BA, Groove MJ, Gangadharam PRJ. 2004. In vitro anti-mycobacterial activities of tree species on cola plant extacts (Sterculiaceae). Phytotherapy Research, 18(5): 414-418. . DOI: http://doi.org/10.1002/ptr.1468

Adeogun II, Fawibe OO, Ajiboye AA, Agboola DA. 2014. Ethnobotanical Survey of Medicinal Plants Used In the Treatment of Skin Diseases in Abeokuta South Local Government of Ogun State Nigeria, Asian Journal of Pharmaceutical Technology \& Innovation, $\quad \mathbf{2 ( 8 )}$ :1-14. DOI: http://doi.org/10.15580/gjbs.2014.2.0103 14006

Adjakpa JB, Akpo LE 2008. Flore ligneuse du bas-delta de l'ouémé dans le Sud-Bénin. Journal des Sciences, 8(4): 1-14. http://www.ucadjds.org 
Adjakpa JB, Yedomonhan H, Ahoton LE, Weesie PDM, Akpo LE. 2013. Structure et diversité floristique des îlots de forêts riveraines communautaires de la Basse vallée de la Sô au Sud-Est du Bénin. Journal of Applied Biosciences, 65: 4902-4913.

Adjakpa JB, Dassoundo HA, Yedomonhan H, Weesie PDM, Akpo LE. 2011. Diversité du peuplement ligneux d'une forêt dense en zone sub-humide : Cas de la forêt de Sakété dans le sud-Bénin en Afrique de l'Ouest. International Journal of Biological and Chemical Sciences, 5(6): 2291-2305. DOI: http://dx.doi.org/10.4314/ijbcs.v5i6.10

Adjanohoun EJ, Adjakidje V, Ahyi MRA, Ake Assi L, Akoegninou A, d'Almeida J, Akpovo F, Bouke K, Chadare M, Cusset G, Dramane K, Eyme J, Gassita J-N, Gbaguidi N, Goudoté E, Guinko S, Hougnon P, Issa L O, Keita A, Kiniffo HV, Kone-Bamba D, MusampaNseyya A, Saadou M, Sodogandji Th, de Souza S, Tchabi A, Zinsou Dossa C, Zohoun Th. 1989. Contribution aux Etudes Ethnobotaniques et Floristiques en République Populaire $d u$ Bénin. Médecine Traditionnelle et Pharmacopée. ACCT: Paris.

Adomou AC, Yedomonhan H, Djossa B, Legba SI, Oumorou M, Akoegninou A. 2012. Etude Ethnobotanique des plantes médicinales vendues dans le marché d'Abomey-Calavi au Bénin. International Journal of Biological and Chemical Sciences, 6(2): 745-772. DOI: http://dx.doi.org/10.4314/ijbcs.v6i2.18

Adomou AC. 2005. Vegetation pattern and environmental gradients in Benin: Implications for biogeography and conservation. $\mathrm{PhD}$ thesis. Wageningen University, Wageningen. p. 133.

Ajayi IO, Ojelere OO. 2013. Chemical composition of ten medicinal plant seeds from South-west Nigeria, Advances in Life Science and Technology, 10: 25-32

Akinloye AJI, lloh HC 1, Olagoke OA. 2012. Significance of Wood Anatomical Features to the Taxonomy of Five Cola
Species. Sustainable Agriculture

Research, 1(2): 21-26.

DOI:10.5539/sar.v1n2p21

Akoègninou A, Van der burg WJ, Van der maesen LJG, Adjakidjè V, Essou JP, Sinsin B, Yédomonhan H. 2006. Flore Analytique $d u$ Bénin. Backhuys Publishers: Leiden, the Netherlands.

Alaribe CSA, Coker H. 2014. Some selected under-utilized edible plants from SouthEast Nigeria used in the treatment of infectious diseases. International Journal of Infectious Diseases, 21:1-460. DOI: http://dx.doi.org/10.1016/j.ijid.2014.03.7 38

Alohou EC, Gbemavo DSJC, Mensah S, Ouinsavi C. 2017. Fragmentation of Forest Ecosystems and Connectivity Between Sacred Groves and Forest Reserves in Southeastern Benin, West Africa. Tropical Conservation Science, 10:1-11.

DOI: 10.1177/194008291773173

Amontcha M, Lougbégnon T, Cledjo PFGA, Ogouwalé E. 2011. Diversité et exploitation des ressources végétales de la réserve naturelle de l'arrondissement de Zinvié. In: Actes du Troisième Colloque des Sciences, Cultures et Technologies de l'UAC-Bénin, 1: 183204.

Amoussou AGO. 2005. Ressources alimentaires végétales de la forêt classée de Niaouli (Sud Bénin): caractérisation et mode de valorisation des produits, thèse d'Ingénieur Agronome, Université Abomey-Calavi, Bénin, p.86.

Anglaaere LCN, Cobbina J, Morag FLS, McDonald A. 2011. The effect of land use systems on tree diversity: farmer preference and species composition of cocoa-based agroecosystems in Ghana. Agroforest Syst., 81: 249-265. DOI: 10.1007/s10457-010-9366-z

Aoudji AKN, Ganglo CJ. 2006. Phytosociologie appliquée à l'aménagement des forêts : cas du périmètre forestier de Pahou (Département de l'Atlantique, Sud- 
Bénin). Journal de Botanique de la Société Botanique de France, 34(3-5): 89-92.

DOI :

http://doi.org/10.1080/12538078.2005.10 515500

Arbonnier M. 2004. Tree, Shrub and Lianas of West Africa Dry Zones. CIRAD, MAGRAF Pub.

Asase A, Tetteh DA. 2010. The role of complex agroforestry systems in the conservation of forest tree diversity and structure in southeastern Ghana. Agroforest Syst., 79: 355-368. DOI: 10.1007/s10457-010-9311-1

Assogbadjo AE, Glèlè Kakaï R, Vodouhê FG, Djagoun CAMS, Codjia JTC, Sinsin B. 2012. Biodiversity and socioeconomic factors supporting farmers' choice of wild edible trees in the agroforestry systems of Benin (West Africa). Forest Policy and Economics, 14: 41-49. DOI: http://doi.org/10.1016/j.forpol.2011.07.0 13

Assogbadjo AE, Adomou A, Lougbégnon T, Fandohan B, Sinsin B. 2011. Réalisation de la monographie des sites identifiés d'aire de conservation communautaire de la biodiversité et élaboration de la stratégie du gel du foncier. Annexe 2, volet biodiversite \& conservation. p.54.

Atato A, Wala K, Batawila K, Lamien N, Akpagana K. 2011. Edible Wild Fruit Highly Consumed during Food Shortage Period in Togo: State of Knowledge and Conservation Status. Journal of Life Sciences, 5: 1046-1057.

Atato A, Wala K, Batawila K, Woegan AY, Akpagana K. 2010. Diversité des fruitiers ligneux spontanés du Togo. Fruit, Vegetable and Cereal Science and Biotechnology, 4(1): 1-9.

Avocèvou-Ayisso C, Sinsin B, Adégbidi A, Dossou G, Damme PV. 2009. Sustainable use of non-timber forest products: Impact of fruit harvesting on Pentadesma butyracea regeneration and financial analysis of its products trade in Benin. Forest Ecology and Management, 257: 1930-1938.

DOI: 10.1016/j.foreco.2009.01.043.
Bascom WR. 1951. Yoruba Food. Africa: Journal of the International African Institute, 21(1): 41-53.

Bello MO, Falade OS, Adewusi SRA, Olawore NO. 2008. Studies on the chemical compositions and antinutrients of some lesser known Nigeria fruits, African Journal of Biotechnology, 7(21): 3972-3979

Biaou SSH. 1999. Etude des possibilités d'aménagement de la forêt classée de Bassila: Structure, dynamique des principaux groupements végétaux et périodicité d'Exploitation. Thèse d'Ingénieur Agronome. FSA/UAC, Abomey Calavi, Bénin. p. 194.

Borokini FB, Abitogun A, Olumayede EG. 2014. Nutritional, Antinitritional and Antimicrobial Activities of Seed and Pulp of Cola millenii. Journal of Applied Chemistry, 7(5): 113-118. DOI: http://doi.org/10.9790/57360752113118

Burkill HM. 2004. The Useful Plants of West Tropical Africa (Vol. 6). Royal Botanic Gardens: Kew.

Ceperley N, Montagnini F, Natta A. 2010. Significance of sacred sites for riparian forest conservation in Central Benin. Bois et Forêt des Tropiques, 303(1): 524.

Codjia JTC, Assogbadjo AE, Ekué MR. 2003. Diversité et valorisation au niveau local des ressources végétales forestières alimentaires du Bénin. Cahier d'Etudes et de Recherches Francophones/Agriculture, 12(5) : 321331.

Dan C. 2009. Etudes écologique, floristique, phytosociologique et ethnobotanique de la forêt marécageuse de Lokoli; Zogbodomey-Bénin. Thèse de Doctorat unique, Faculté des Sciences, Université Libre de Bruxelles (ULB), Bruxelles Belgique, p.260.

Dan Guimbo I, Barage M, Douma S. 2012. Etudes préliminaires sur l'utilisation alimentaire des plantes spontanées dans les zones périphériques du parc $\mathrm{W}$ du Niger. Int. J. Biol. Chem. Sci., 6(6): 
4007-4017.

DOI:

http://dx.doi.org/10.4314/ijbcs.v6i6.12.

Dansi A, Adjatin A, Adoukonou-Sagbadja H, Faladé V, Adomou AC, Yedomonhan H, Akpagana K, de Foucault B. 2009. Traditional leafy vegetables in Benin: folk nomenclature, species under threat and domestication. Acta Botanica Gallica, 156(2): 183-199. . DOI: http://doi.org/10.1080/12538078.2009.10 516150

Dansi A, Adjatin A., Adoukonou-Sagbadja H, Faladé V, Yedomonhan H, Odou D, Dossou B. 2008. Traditional leafy vegetables and their use in the Benin Republic. Genetic Resources and Crop Evolution, 55: 1239-1256. DOI: http://doi.org/10.1007/s10722-008-9324z

Dansi AA, Adomou AC, Adeoti K. 2010. Culture des légumes feuilles traditionnels. In Atlas de la Biodiversité de l'Afrique de l'Ouest: Bénin (Tome I), Sinsin B, Kampmann D (eds). Cotonou et Frankfurt/Main; 234-247.

De Souza S. 2008. Flore du Bénin (tome 3) : Noms des Plantes dans les Langues Nationales Béninoises (2 ${ }^{\text {ème }}$ édition). Imprimerie Tundé: Cotonou.

Denou A, Koudouvo K, Togola A, Aziati KY, Esseh K, Ajavon CA, Essien K, Aklikobou K, Sanogo R, Diallo D, Gbeassor M. 2016. Traditionnel knowledge on antimalarial plants having analgesic properties used in Togo Maritime Region. The Journal of Ethnobiology and Traditional Medicine. Photon, 126 : 1160-1170.

Dossou ME, Houessou GL, Lougbégnon OT, Tenté AHB, Codjia JTC. 2012. Etude ethnobotanique des ressources forestières ligneuses de la forêt marécageuse d'Agonvè et terroirs connexes au Bénin. Tropicultura, 30(1) : 41-48.

Dougnon TV, Déguénon E, Fah L, Lègba B, Hounmanou YMG, Agbankpè J, Amadou A, Koudokpon1 H, Fabiyi K, Aniambossou A, Assogba P, Hounsa E, de Souza M, Avlessi F, Dougnon TJ, Gbaguidi F, Boko M, Bankolé HS,
Baba-Moussa L. 2017. Traditional treatment of human and animal salmonelloses in Southern Benin: Knowledge of farmers and traditherapists. Veterinary World, 10(6): 580-592.

DOI: 10.14202/vetworld.2017.580-592

FAO. 2004. Forest Genetic Resources Conservation and Management. Vol. 1: Ovierview, Concepts and Systematic Approaches. International Plant Genetic Resources Institute: Italy, Rome.

Ganglo JC, de Foucault B. 2006. Plant communities, forest site identification and classification in Toffo reserve, South-Benin. Bois et Forêts des Tropiques, 288(2): 25-38.

Giwa OE. 2012. Phytochemical and antimicrobial properties of seed and pulp of monkey cola (cola millenii) on some selected clinical and food borne isolate. International Journal of Applied Biology, 3(3): 390-400.

Gouwakinnou GN, Lykke AM, Assogbadjo AE, Sinsin B. 2011. Local knowledge, pattern and diversity of use of Sclerocarya birrea. Journal of Ethnobiology and Ethnomedicine, 7(8): 1-9.

DOI: http://dx.doi.org/10.1186/1746-4269-7-8

Hèdégbètan GC. 2011. Etude écologique de l'îlot forestier Bahazoun dans la Vallée $d u$ Sitatunga, arrondissement de Kpanroun (Commune d'AbomeyCalavi), Bénin. Mémoire de Licence Professionnelle, Université d'AbomeyCalavi, Bénin, p.50.

Hermans M, Akoègninou A, Van der Maesen LJG. 2004. Medicinal plants used to treat malaria in southern Benin. Economic Botany, 58(sp1): 239-252. DOI: doi.org/10.1663/0013-0001 (2004)58[S239:MPUTTM]2.0.CO;2

Houenon JG. 1980. Etudes pharmacologiques préliminaires d'un diurétique: Cola millenii. Mémoire du diplôme d'études de technicien supérieur, CPU/UNB, Abomey-Calavi, p.31.

Houessou LG, Lougbegnon TO, Gbesso FGH, Anagonou LES, Sinsin B. 2012. Ethno- 
botanical study of the African star apple (Chrysophyllum albidum G. Don) in the Southern Benin (West Africa). Journal of Ethnobiology and Ethnomedicine, 8(40): $1-10 . \quad$ DOI: http://doi.org/10.1186/1746-4269-8-40

Houètchégnon T, Gbèmavo DSJC, Ouinsavi C, Sokpon N. 2015. Ethnobotanical knowledge and traditional management of mesquite (Prosopis africana Guill., Perrot. et Rich.) populations in Benin, West Africa. The Journal of Ethnobiology and Traditional Medicine. Photon, 125: 1124-1135.

Houmenou V, Adjatin A, Tossou MG, Yedomonhan H, Dansi A, Gbenou J, Akoegninou A. 2017. Etude ethnobotanique des plantes utilisées dans le traitement de la stérilité féminine dans les départements de l'Ouémé et du plateau au Sud Bénin. International Journal of Biological and Chemical Sciences, 11(4): 1851-1871. DOI: http://dx.doi.org/10.4314/ijbcs.v11i4.34

Hutchinson J, Dalziel JM, 1958. Flora of West Tropical Africa (2nd edn, Vol 1, Part 2). Royal Botanic Garden: Kew.

Itoandon EE, Nwachukwu VA, Erukainure OL, Lasore OO, Nwagala PN. 2016. Cola millenii Leaf Ethyl Acetate Extract: Fourier Transform Infrared (FTIR) Spectroscopy; Modulatory Effect on Serum Indices and Redox Biomarkers. European Pharmaceutical Journal, 2: 711. DOI: $10.1515 /$ afpuc-2016-0016

Jagoret P, Michel- Dounias I, Snoeck D, Ngnogue HT, Malezieux E. 2012. Afforestation of savannah with cocoa agroforestry systems: a small-farmer innovation in central Cameroon. Agroforest Syst., 86: 493-504. DOI: 10.1007/s10457-012-9513-9

Kakpo SB. 2013. Caractéristiques Structurales et Ecologiques des Forêts de Bonou et d'Itchèdè au Sud-Est du Benin. Thèse d'Ingénieur Agronome, Faculté des Sciences Agronomiques, Université d'Abomey-Calavi, Bénin, p.70.

Kakpo SB, Yehouenou Tessi DR, Gbetoho AJ, Aoudji AKN, Ganglo JC. 2018.
Répartition spatiale de Cola millenii $\mathrm{K}$. Schum., Dialium guineense Wild. Et Afzelia africana Smith ex Pers. dans les forêts secondaires du Sud Bénin (Afrique de l'Ouest). Int. J. Biol. Chem. Sci., 12(1): 353-362. DOI : https://dx.doi.org/10.4314/ijbcs.v12i1.28

Kokou K, Batawila K, Akpagana K, Tossou MG, Akoegninou A. 2013. Impact écologique de la cueillette et commercialisation des plantes médicinales $\mathrm{au}$

Togo.www.glinus.com/2013/12/impactecologique-de-la-cueillette-et.html. Consulté le 16/09/2017

Koudouvo K, Esseh K, Denou A, Aziati T, Ajavon C, Afanyibo Y-G, Agbonon A, Sanogo R, Dougnon J, Aklikokou K, Aguiyi JC, Diallo D, Mensah GA, Gbeassor M. 2016. Etude ethnopharmacologique des recettes médicinales antipaludiques du Togo pour la formulation d'un phytomédicament de prise en charge du paludisme. Bulletin de la Recherche Agronomique du Bénin, 79: 54-70.

Koulibaly A, Nicaise A, Diomandé M, Konaté I, Traoré D, Bill R, Kouadio YJ. 2016. Conséquences de la culture de l'anacardier (Anacardium occidentale L.) sur les caractéristiques de la végétation dans la région du Parc National de la Comoé (Côted'Ivoire). International Journal of Innovation and Applied Studies, 17(4): 1416-1426.

Kpodar MS, Karou SD, Katawa, G, Anani K, Gbekley HE. Adjrah Y. Tchacondo T, Batawila K, Simpore J. 2016. An ethnobotanical study of plants used to treat liver diseases in the Maritime region of Togo. Journal of Ethnopharmacology, 181: 263-273. DOI:

http://dx.doi.org/10.1016/j.jep.2015.12.0 51

Laleye OAF, Ahissou H, Olounlade AP, Azando EVB, Laleye A. 2015. Etude bibliographique de trois plantes antidiabétiques de la flore béninoise: Khaya senegalensis (Desr) A. Juss 
(Meliaceae), Momordica charantia Linn (Cucurbitaceae) et Moringa oleifera Lam (Moringaceae) : International Journal of Biological and Chemical Sciences, 9(5): 2682-2700.

DOI: http://dx.doi.org/10.4314/ijbcs.v9i5.38

Makalao MM, Savadogo A, Zongo C, Traore AS. 2015. Composition nutritionnelle de 10 fruits sauvages consommés dans trois départements du Tchad. International Journal of Biological and Chemical Sciences, 9(5): 2385-2400. DOI : http://dx.doi.org/10.4314/ijbcs.v9i5.11

Morakinyo JA. 1995. Gene Exchange between Cola millenii and Cola nitida: Hybridization and Hybrid Seed Viability. Klobex Academic Publishers, 151-153.

Motte E. 1980. Les plantes chez les Pygmées Aka et les Monzombo de la Lobaye (Centrafrique) : Contribution à une Etude Ethnobotanique Comparative chez des Chasseurs-Cueilleurs et des Pêcheurs-Cultivateurs Vivant dans un même Milieu Végétal. Agence de Coopération Culturelle et Technique: Paris

Muoghalu JI, Okeesan OO. 2005. Climber species composition, abundance and relationship with trees in a Nigerian secondary forest. African Journal of Ecology, 43: 258-266.

Natta AK. 2003. Ecological assessment of riparian forest in Benin: phytodiversity, phytosociology and spatial distribution of tree species. PhD Thesis, Wageningen Univ., Netherlands, p.222.

Ndah NR, Egbe AE, Bechem E, Asaha S, Yengo T, Chia EL, Eyenieh NM. 2013. Ethnobotanical study of commonly used medicinal plants of the Takamanda Rainforest South West, Cameroon. African Journal of Plant Science, 7(1): 21-34.

N'dri MTK, Gnahoua MG, Konan EK, Traoré D. 2008. Plantes alimentaires spontanées de la région du Fromager (Centre Ouest de la Côte d'Ivoire) flore, habitats et organes consommés. Sci. \& Nat., 5: 6170.

DOI:

http://doi.org/10.4314/scinat.v5i1.42152
Nkongmeneck B-A. 1982. Contribution à l'étude du genre Cola au Cameroun. Thèse de doctorat en sciences biologiques. Faculté des sciences, Université de Yaoundé, Cameroun, p. 198 .

Nyadanu DL, Aboagye M, Akromah R, Dansi A, 2016. Agro-biodiversity and challenges of on-farm conservation: the case of plant genetic resources of neglected and underutilized crop species in Ghana. Genet Resour Crop Evol., 63:1397-1409. DOI 10.1007/s10722015-0327-2

Odugbemi T. 2006. Outlines and Pictures of Medicinal Plants from Nigeria (Vol.10). University of Lagos Press: Lagos

Oladokun MAO. 1986. Vegetative propagation studies in kola (cola spp.). II. Soil layering and budding. Cacao Research Institute of Nigeria, Ibadan (Nigeria). http://www.agris.fao.org

Olayiwola IO, Akinfenwa VO, Oguntona CO, Sanni SA, Onabanjo OO, Afolabi, WAO. 2013. Phytonutrient, Antioxidant and Mineral Composition of Some Wild Fruits in South West Nigeria. Nigeria Food Journal, 31(2): 33-40.

Orisakeye OT, Ojo AA. 2013. Antimicrobial and antioxidant evaluation of various parts of Cola milleni K. Schum plant. African Journal of Pharmacy and Pharmacology, 7(48): 3019-3025. DOI 10.5897/AJPP2013.3416

Osipitan AA, Odebiyi JA, Lawal OA, Somade AA. 2010. Evaluation of the extracts of some tropical plants in the management of Prostephanus truncatus (Horn) infestation in maize (Zea mays L.). Archives of Phytopathology and Plant Protection, 43(10): 1001-1010 DOI: 10.1080/03235400802214877

Oumorou M. 2003. Etudes écologique, floristique, phytogéographique et phytosociologique des inselbergs du Bénin. Thèse de doctorat en sciences. Université Libre de Bruxelles, Belgique, p.216.

Oyemitan IA, Kolawole F, Abass L, Oyedeji AO. 2016. Neuropharmacological 
Activities of Ethanolic Extract of Cola millenii Dried Leaf in Rats. European Journal of Medicinal Plants, 16(2): 1-12 DOI:

http://doi.org/10.9734 /ejmp/2016/2871 8

Pascual-Garrido A, Buba U, Nodza G, Sommer V. 2012. Obtaining Raw Material: Plants as Tool Sources for Nigerian Chimpanzees. Folia Primatol, 83: $24-44$. DOI: $10.1159 / 000338898$.

Pujol R. 1957. Etude préliminaire des principaux insectes nuisibles aux Colatiers. Journal d'Agriculture Traditionnelle et de Botanique Appliquée, 4(5-6): 241-264.

Ratsch C. 2005. The Encyclopedia of Pscychoactive Plants: Ethnopharmacology and its Applications. Rochester: Vermont

Russell TA. 1955. The kola of Nigeria and the Cameroon. Agriculture Tropicale, Trin, 32(3): 211-277.

Sangare MM, Sina H, Dougnon J, Bayala B, Ategbo J-M, Dramane KL. 2012. Etude ethnobotanique des plantes hépatotropes et de l'usage traditionnel de Gomphrena celosioides Mart. (Amaranthaceae) au Bénin. International Journal of Biological and Chemical Sciences, 6(6): 5008-5021.

DOI: http://dx.doi.org/10.4314/ijbcs.v6i6.20

Sonibare MA, Soladoye MO, Esan OO, Sonibare OO. 2009. Phytochemical and Antimicrobial Studies of Four Species of Cola Schott \& Endl. (Sterculiaceae). Afr $J$ Tradit Complément Altern Med., 6(4): 518-525.

DOI: http://doi.org/10.4314/ajtcam.v6i4.57182
Sourou BN, Yabi J, Ouinsavi CAIN, Sokpon N. 2016. Importance socio-économique de la prune rouge (Haematostaphis barteri Hook F.) au Bénin. International Journal of Biological and Chemical Sciences, 10(1): 326-343. DOI: http://dx.doi.org/10.4314/ijbcs.v10i1.25

Ubon JA, Akpanabiatu MI, Akpanyung EO, Ufot UF. 2017. Effects of ethanolic extracts of Cola millenii K. Schum seed on biochemical and toxicological indices of male wistar albino rats. Journal of Pharmacognosy and Phytochemistry, 6(1): 160-166.

Woegan YA, Akpavi S, Dourma M, Atato A, Wala K, Akpagana K. 2013. Etat des connaissances sur la flore et la phytosociologie de deux aires protégées de la chaîne de l'Atakora au Togo : Parc National Fazao-Malfakassa et Réserve de Faune d'Alédjo. International Journal of Biological and Chemical Sciences, 7(5): 1951-1962. DOI: http://dx.doi.org/10.4314/ijbcs.v7i5.14

Yakubu FB, Adejoh OP, Ogunade JO, Igboanugo ABI. 2014. Vegetative Propagation of Garcinia kola (Heckel). World Journal of Agricultural Sciences, 10(3): $\quad 85-90 . \quad$ DOI: 10.5829/idosi.wjas.2014.10.3.1808

Yédomonhan $\mathrm{H}$, Hounadagba CJ, Akoègninou A, van der Maesen LJG. 2008. Structure et diversité floristique de la végétation des inselbergs du secteur méridional du Centre-Bénin. Systematic and Geography of Plants, 78: 111-125. 